\title{
NICOLA ORRÙ \\ On a weakly hyperbolic equation with a term of order zero
}

Annales de la faculté des sciences de Toulouse $6^{e}$ série, tome $6, \mathrm{n}^{\circ} 3$ (1997), p. 525-534

<http://www.numdam.org/item?id=AFST_1997_6_6_3_525_0>

(C) Université Paul Sabatier, 1997, tous droits réservés.

L'accès aux archives de la revue «Annales de la faculté des sciences de Toulouse » (http://picard.ups-tlse.fr/ annales/) implique l'accord avec les conditions générales d'utilisation (http://www.numdam.org/conditions). Toute utilisation commerciale ou impression systématique est constitutive d'une infraction pénale. Toute copie ou impression de ce fichier doit contenir la présente mention de copyright.

\section{NumDam}

Article numérisé dans le cadre du programme

Numérisation de documents anciens mathématiques

http://www.numdam.org/ 


\title{
On a weakly hyperbolic equation with a term of order zero ${ }^{(*)}$
}

\author{
Nicola OrRÙ (1)
}

RÉSUMÉ. - Nous étudions une équation faiblement hyperbolique du second ordre, en supposant que les coefficients de la partie principale sont fonctions analytiques qui ne dépendent que du temps. Nous démontrons que, en ajoutant un terme d'ordre zéro $c(t, x) u$ avec $c \in C\left(\mathbb{R}^{n+1}\right)$, le problème de Cauchy reste bien posé dans $C^{\infty}$.

ABstract. - We study a weakly hyperbolic equation of second order, supposing that the coefficients of the principal part are analytic and depend only on time. We prove that if we add a term of order zero $c(t, x) u$, with $c \in C\left(\mathbb{R}^{n+1}\right)$, the Cauchy problem remains well-posed in $C^{\infty}$.

\section{Introduction}

In the literature many authors study the Cauchy problem in $C^{\infty}$ for a weakly hyperbolic equation of second order

$$
u_{t t}-\sum_{i j} a_{i j}(t) u_{x_{i} x_{j}}=0
$$

with

$$
a(t, \xi)=\sum a_{i j}(t) \xi_{i} \xi_{j} \geq 0 \quad\left(\xi \in \mathbb{R}^{n}\right)
$$

In [CJS] it is studied the case of analytic coefficients $a_{i j}(t)$ in $[0, T]$, showing that the Cauchy problem is well-posed. The same method works

(*) Reçu le 17 juillet 1992

(1) Università di Sassari, Istituto di Matematica e Fisica, via Vienna, 2, I-07100 Sassari (Italia) 
when we add a term of order zero $c(t) u$ to the equation, with the coefficient $c(t)$ in $L^{1}(0, T)$. It follows from the proof of the theorem in [CJS] that the same conclusion holds if $a_{i j} \in C^{1}([0, T])$ and satisfy the logarithmic condition.

$$
\int_{0}^{T} \frac{\left|a_{t}^{\prime}(t, \xi)\right|}{a(t, \xi)+1} \mathrm{~d} t \leq N \log (1+|\xi|)+C, \quad \forall \xi \in \mathbb{R}^{n} .
$$

Oleinik [Ol] studies equations of the type:

$$
u_{t t}-\sum_{i j}\left(a_{i j}(t, x) u_{x_{i}}\right)_{x_{j}}+c(t, x) u=0,
$$

supposing that $a_{i j}(t, x), c(t, x)$ are of $C^{\infty}$ class in $[0, T] \times \mathbb{R}^{n}$, satisfying (2) and

$$
\sum_{i j} a_{i j, t}^{\prime}(t, x) \xi_{i} \xi_{j}+C \sum_{i j} a_{i j}(t, x) \xi_{i} \xi_{j} \geq 0
$$

or

$$
-\sum_{i j} a_{i j, t}^{\prime}(t, x) \xi_{i} \xi_{j}+(\alpha(T-t))^{-1} \sum_{i j} a_{i j}(t, x) \xi_{i} \xi_{j} \geq 0 .
$$

Then there is also well-posedness in $C^{\infty}$. If $a_{i j}(t)$ are analytic, Oleinik conditions (5), (5') may fail to hold, for example take $a(t, \xi)=\left(\xi_{1}-t \xi_{2}\right)^{2}$.

Kajitani [Ka], in a recent paper, considers the equation

$$
P u=\left[\partial_{t}^{2}-\sum_{i j} a_{i j}(t, x) \partial_{x_{i}} \partial_{x_{j}}+\sum_{j} a_{j}(t, x) \partial_{x_{j}}\right] u=0,
$$

with coefficients in $C^{1}\left([0, T] ; C^{\infty}\right)$.

Posing $\langle\xi\rangle^{2}=1+\sum_{j} \xi_{j}^{2}$, he assumes (2) and that there is $f(t, x, \xi) \in$ $C^{\infty}\left(\mathbb{R} \times \mathbb{R}^{n} \times \mathbb{R}^{n}\right)$ such that

$$
\begin{array}{r}
\frac{\left|a_{t}^{\prime}(t, x, \xi)\right|}{a(t, x, \xi)+(f(t, x, \xi))^{2}}+\frac{\left|\sum_{j} a_{j}(t, x) \xi_{j}\right|}{\sqrt{a(t, x, \xi)+(f(t, x, \xi))^{2}}} \leq f(t, x, \xi), \\
\Lambda(t, x, \xi)=\int_{0}^{t} f(s, x, \xi) \mathrm{d} s \leq C(\log \langle\xi\rangle+1), \\
\left|f_{(\beta)}^{(\alpha)}(t, x, \xi)\right| \leq C_{\alpha, \beta}(f(t, x, \xi))^{1+|\beta|}\langle\xi\rangle^{-|\alpha|}, \quad \forall \alpha, \forall \beta,|\beta|<1, \\
\exists \delta>0 \text { s.t. } \quad \Lambda_{t}^{2}-a\left(t, x, \Lambda_{x}\right) \geq \delta\left|\left(\Lambda_{t}, \Lambda_{x}\right)(t, x, \xi)\right|^{2}, \\
(t, x) \in[0, T] \times \mathbb{R}^{n}, \xi \in \mathbb{R}^{n} .
\end{array}
$$


On a weakly hyperbolic equation with a term of order zero

Then he proves that the Cauchy problem for (6) is well-posed in $C^{\infty}$. The same result should be true in the presence of a term of order zero.

In this paper we shall study again the hyperbolic equation (4)

$$
u_{t t}-\sum_{i j} a_{i j}(t) u_{x_{i} x_{j}}+c(t, x) u=0
$$

with analytic coefficients $a_{i j}(t)$ satisfying (2) and with the coefficient $c(t, x) \in C\left([0, T] ; C^{\infty}\right)$ depending also on space variables. We prove that there is $C^{\infty}$ well-posedness. We observe that there exist analytic coefficients $a_{i j}(t)$ which don't verify Kajitani conditions (see the appendix). We show the $C^{\infty}$ well-posedness also if $a(t, \xi)=a(t) \sum \xi_{j}^{2}$, with $a(t) \in C^{1}([0, T])$, satisfying (2) and (3). We don't know if this is still true for a generic quadratic form $a(t, \xi)=\sum_{i j} a_{i j}(t) \xi_{i} \xi_{j}$.

\section{The logarithmic condition}

Let $a_{i j}(t)$ be analytic functions on $[0, T]$, satisfying $(0.2)$.

We have the following results (stated without proof in [CJS]).

Lemma 1. - The function

$$
t \mapsto a_{t}^{\prime}(t, \xi)
$$

vanishes identically or has at most $m$ roots in $[0, T]$ for every $\xi \in \mathbb{R}^{n}$, where $m$ is an integer independent of $\xi$.

Proof. - Lemma 1 is a consequence of the following statement:

" if $a_{1}(t), \ldots, a_{k}(t)$ are real analytic functions on $[0, T]$, there exists an integer $m$ such that every linear combination of $a_{1}(t), \ldots, a_{k}(t)$, which is different from zero, has at most $m$ roots."

If $a_{1}(t), \ldots, a_{k}(t)$ are linearly dependent we can eliminate some of them obtaining an independent set. So we can suppose that $a_{1}(t), \ldots, a_{k}(t)$ are linearly independent.

It is enough to prove that for every $t \in[0, T]$ there exists a neighbourhood $U$ ot $t$ and an integer $p$ such that every linear combination of $a_{1}, \ldots, a_{k}$ has at most $p$ zeroes in $U$. The thesis follows from the compactness of $[0, T]$. 
Let us suppose that there exists a number $t_{0} \in[0, T]$ such that for every $p$ there exists a linear combination $b_{p}(t)=\alpha_{1, p} a_{1}+\cdots+\alpha_{k, p} a_{k}$ with at least $p$ isolated zeroes in $\left|t-t_{0}\right|<1 / p$ and let us derive a contradiction.

We can suppose that $\alpha_{1, p}^{2}+\cdots+\alpha_{k, p}^{2}=1 \forall p$ and that $\alpha_{1, p} \rightarrow$ $\alpha_{1,0}, \ldots, \alpha_{k, p} \rightarrow \alpha_{k, 0}$ for $p \rightarrow+\infty$ with $\alpha_{1,0}^{2}+\cdots+\alpha_{k, 0}^{2}=1$, so that $b_{p}(t) \rightarrow b_{0}(t)=\sum_{j=0}^{k} \alpha_{j, 0} a_{j}(t)$ uniformly together with all the derivatives.

We have that $b_{p}^{(h)}(t)$ vanishes in a point $t_{p, h}$, with $\left|t_{p, h}-t_{0}\right|<1 / p$ for $h=0, \ldots, p-1$. Hence

$$
b_{0}^{(h)}\left(t_{0}\right)=\lim _{p \rightarrow \infty} b_{p}^{(h)}\left(t_{p, h}\right)=0 .
$$

So $b_{0}(t) \equiv 0$, contradicting the hypothesis that $a_{1}(t), \ldots, a_{k}(t)$ are linearly independent.

Lemma 2.- There exist positive constants $C, N$ such that (0.3) is verified.

Proof.- By lemma 1, after fixing $\xi$, there are $2 k+2$ points

$$
0=s_{0} \leq t_{0} \leq s_{1} \leq t_{1} \leq \cdots \leq s_{k} \leq t_{k}=T
$$

such that $a(s, \xi)$ is decreasing for $s_{j} \leq s \leq t_{j}$ and increasing for $t_{j} \leq s \leq$ $s_{j+1}$.

We can choose $k \leq m / 2+1$. We have

$$
\begin{aligned}
\int_{0}^{T} & \frac{\left|a_{t}^{\prime}(s, \xi)\right|}{a(s, \xi)+1} \mathrm{~d} s= \\
\quad & \sum_{j=0}^{k} \int_{s_{j}}^{t_{j}} \frac{-a_{t}^{\prime}(s, \xi)}{a(s, \xi)+1} \mathrm{~d} s+\sum_{j=0}^{k-1} \int_{t_{j}}^{s_{j+1}} \frac{a_{t}^{\prime}(s, \xi)}{a(s, \xi)+1} \mathrm{~d} s= \\
& =\sum_{j=0}^{k} \log \frac{a\left(s_{j}, \xi\right)+1}{a\left(t_{j}, \xi\right)+1}+\sum_{j=0}^{k-1} \log \frac{a\left(s_{j+1}, \xi\right)+1}{a\left(t_{j}, \xi\right)+1} \leq \\
& \leq(2 k+1) \log \left(C_{0} \xi^{2}+1\right) \leq \\
& \leq 2(m+3) \log (|\xi|+1)+(m+3) \log \left(C_{0}+1\right)
\end{aligned}
$$

where $C_{0}=\sup \{a(t, \xi):|\xi|=1,0 \leq t \leq T\}$. 


\section{Existence theorem}

THEOREM .- We consider the equation

$$
u_{t t}-\sum_{i j} a_{i j}(t) u_{x_{i} x_{j}}+c(t, x) u=0
$$

with $a_{i j}(t)$ real analytic functions on $[0, T]$, fulfilling (0.2). Let $c(t, x)$ be a function in $C\left([0, T] ; C^{\infty}\left(\mathbb{R}^{n}\right)\right)$.

Then the Chauchy problem for equation (1) is well-posed.

Proof. - By the finite speed of propagation we can suppose $c(t, x) \epsilon$ $C\left([0, T] ; C_{0}^{\infty}\left(\mathbb{R}^{n}\right)\right)$. Let us define the energy

$$
E(t, \xi)=\left(\left|v^{\prime}\right|^{2}+(a(t, \xi)+1)|v|^{2}\right) k(t, \xi),
$$

where $v=\mathcal{F}_{x} u$,

$$
k(t, \xi)=\exp \left(-\int_{0}^{t} \frac{\left|a_{t}^{\prime}(s, \xi)\right|}{a(s, \xi)+1} \mathrm{~d} s\right)\left(1+\xi^{2}\right)^{\sigma}
$$

and $\sigma$ is a real number. Integrating we have:

$$
\begin{aligned}
\mathcal{E}(t) & =\int E(t, \xi) \mathrm{d} \xi=\int\left[\left|v^{\prime}\right|^{2}+(a(t, \xi)+1)|v|^{2}\right] k(t, \xi) \mathrm{d} \xi \\
\mathcal{E}(t) & \leq C \int\left[\left(1+\xi^{2}\right)^{\sigma}\left|v^{\prime}\right|^{2}+\left(1+\xi^{2}\right)^{\sigma+1}|v|^{2}\right] \mathrm{d} \xi \\
\mathcal{E}(t) \geq & \frac{1}{C} \int\left[\left(1+\xi^{2}\right)^{\sigma-N / 2}\left|v^{\prime}\right|^{2}+\left(1+\xi^{2}\right)^{\sigma-N / 2}|v|^{2}\right] \mathrm{d} \xi \\
E_{t}^{\prime}(t, \xi) & =\left(2 \Re v^{\prime \prime} \overline{v^{\prime}}+a_{t}^{\prime}(t, \xi)|v|^{2}+a(t, \xi) 2 \Re v \overline{v^{\prime}}+\right. \\
& \left.\quad+2 \Re v \bar{v}^{\prime}-\frac{\left|a_{t}^{\prime}(t, \xi)\right|}{a(t, \xi)+1}\left|v^{\prime}\right|^{2}-\left|a_{t}^{\prime}(t, \xi)\right||v|^{2}\right) k(t, \xi) .
\end{aligned}
$$

To estimate the integral of the right hand side we need the following result. 
Lemma 3.- The function $k(t, \xi)$, continuous in $\xi \in \mathbb{R}^{n}$, is a temperate weight function [Hör], that is

$$
k(t, \xi+\eta) \leq k(t, \xi) C(1+|\eta|)^{N}, \quad \forall \xi, \eta, \forall t
$$

where $C, N$ are positive constants, which don't depend on $t$.

Proof of the lemma. - It is enough to show that

$$
k_{1}(t, \xi)=\exp \left(\int_{0}^{t} \frac{\left|a_{t}^{\prime}(s, \xi)\right|}{a(s, \xi)+1} \mathrm{~d} s\right)
$$

is a temperate weight function, since

$$
k(t, \xi)=k_{1}(t, \xi)^{-1}\left(1+\xi^{2}\right)^{\sigma},
$$

where $\left(1+\xi^{2}\right)^{\sigma}$ is a temperate weight function, and the inverse and the product of temperate weight functions have the same property. Now we observe that taking $k=[m / 2]+1$ and

$$
0=s_{0} \leq t_{0} \leq s_{1} \leq t_{1} \leq \cdots \leq s_{k} \leq t_{k}=T
$$

we have

$$
\sum_{j=0}^{k} \log \frac{a\left(s_{j}, \xi\right)+1}{a\left(t_{j}, \xi\right)+1}+\sum_{j=0}^{k-1} \log \frac{a\left(s_{j+1}, \xi\right)+1}{a\left(t_{j}, \xi\right)+1} \leq \exp \left(\int_{0}^{t} \frac{\left|a_{t}^{\prime}(s, \xi)\right|}{a(s, \xi)+1} \mathrm{~d} s\right)
$$

and the equality holds for suitable values of $s_{j}, t_{j}$. Hence

$$
\begin{aligned}
k_{1}(t, \xi) & =\sup \left\{\prod_{j=0}^{k} \frac{a\left(s_{j}, \xi\right)+1}{a\left(t_{j}, \xi\right)+1} \cdot \prod_{j=0}^{k-1} \frac{a\left(s_{j+1}, \xi\right)+1}{a\left(t_{j}, \xi\right)+1}: s_{j}, t_{j} \text { satisfy }(3)\right\} \\
& =\sup k_{\left\{s_{j}\right\},\left\{t_{j}\right\}}(t, \xi) .
\end{aligned}
$$

It remains to show that $k_{\left\{s_{j}\right\},\left\{t_{j}\right\}}(t, \xi)$ is a temperate weight function, with constants $C, N$ which don't depend on $\left\{s_{j}\right\},\left\{t_{j}\right\}$. This follows from the fact that $a(s, \xi)+1$ is a temperate function with constants $C, N$ independent of $s$.

$$
\begin{aligned}
a(s, \xi+\eta)+1 \leq & 2 a(s, \xi)+2 a(s, \eta)+1 \\
\leq & (a(s, \xi)+1) 2(a(s, \eta)+1) \\
\leq & (a(s, \xi)+1) C(1+|\eta|)^{2} \\
& -530-
\end{aligned}
$$


On a weakly hyperbolic equation with a term of order zero

$$
C=2 \max \left(1, \sup _{s,|\xi|=1} a(s, \xi)\right)
$$

Since $k$ is a temperate weight function, we have

$$
\begin{aligned}
& \int|\widehat{c u}|^{2} k(t, \xi) \mathrm{d} \xi= \\
& \quad=\int k(t, \xi)\left|\frac{1}{(2 \pi)^{n}} \int \widehat{c}(\xi-\eta) \widehat{u}(\eta) \mathrm{d} \eta\right|^{2} \mathrm{~d} \xi \leq \\
& \quad \leq \int k(t, \xi) \frac{1}{(2 \pi)^{n}} \int|\widehat{c}(\xi-\eta)||\widehat{u}(\eta)|^{2} \mathrm{~d} \eta \mathrm{d} \xi \frac{1}{(2 \pi)^{n}} \int\left|\widehat{c}\left(\eta_{1}\right)\right| \mathrm{d} \eta_{1} \\
& \quad \leq \frac{1}{(2 \pi)^{n}} \iint k(t, \eta)|\widehat{u}(\eta)|^{2}|\widehat{c}(\xi-\eta)| C(1+|\xi-\eta|)^{N} \mathrm{~d} \eta \mathrm{d} \xi\|\mid\| c \| \\
& \quad \leq \int k(t, \eta)|\widehat{u}(\eta)|^{2} \mathrm{~d} \eta \frac{C}{(2 \pi)^{n}} \int|\widehat{c}(\xi)|(1+|\xi|)^{N} \mathrm{~d} \xi|\|c\|| \\
& \quad \leq\left.\left|\|c\|^{2} \int k(t, \eta)\right| \widehat{u}(\eta)\right|^{2} \mathrm{~d} \eta \leq\|c\|^{2} \mathcal{E}(t) .
\end{aligned}
$$

Hence

$$
\begin{gathered}
\mathcal{E}^{\prime}(t) \leq(2+\|c(t)\| \mid) \mathcal{E}(t) \\
\mathcal{E}(t) \leq \mathcal{E}(0) \exp ((2+\sup \|c(t)\| \mid) t) .
\end{gathered}
$$

This inequality remains true if we substitute $a(t, \xi)$ with $a(t, \xi)+\epsilon \xi^{2}$, with $\epsilon>0$. The corresponding equation, which is strictly hyperbolic, will have a solution of class $C^{\infty}$. If the initial data $u_{0}, u_{1} \in C_{0}^{\infty}\left(B\left(x_{0}, r_{0}\right)\right)$, $x_{0} \in \mathbb{R}^{n}, r_{0}>0$, the solution has support in $B\left(x_{0}, r_{0}+v_{\epsilon} t\right)$ at the time $t$ $\left(v_{\epsilon}^{2}=\sup _{t,|\xi|=1}\left[a(t, \xi)+\epsilon|\xi|^{2}\right]\right)$.

The energy estimates allow to bound the norms in $H^{s}$ of the solution $u^{(\epsilon)}(t, x)$, uniformly with respect to $\epsilon>0$. There will be a solution of the limit problem. The uniqueness can be proved by duality.

Remark. - We don't know if the theorem is true for general $a_{i j}(t)$ of class $C^{1}$ fulfilling $(0.2),(0.3)$. If $a(t, \xi)=a(t) \xi^{2}$ the following lemma permits to apply the theorem. 
LEMMA 4. - If $a(t)$ is of class $C^{1}$ and $a(t, \xi)=a(t) \xi^{2}$ satisfies (0.2), (0.3), then

$$
k(t, \xi)=\exp \left(\int_{0}^{t} \frac{\left|a^{\prime}(s)\right| \xi^{2}}{a(s) \xi^{2}+1} \mathrm{~d} s\right)
$$

is a temperate weight function.

Proof. - We have to verify that

$$
\int_{0}^{t} \frac{\left|a^{\prime}(s)\right|(\xi+\eta)^{2}}{a(s)(\xi+\eta)^{2}+1} \mathrm{~d} s \leq \int_{0}^{t} \frac{\left|a^{\prime}(s)\right| \xi^{2}}{a(s) \xi^{2}+1} \mathrm{~d} s+N \log (1+|\eta|)+C .
$$

It suffices to show that

$$
\begin{gathered}
\int_{0}^{t} \frac{\left|a^{\prime}(s)\right|(|\xi|+|\eta|)^{2}}{a(s)(|\xi|+|\eta|)^{2}+1} \mathrm{~d} s-\int_{0}^{t} \frac{\left|a^{\prime}(s)\right| \xi^{2}}{a(s) \xi^{2}+1} \mathrm{~d} s \leq N \log (1+|\eta|)+C, \\
\int_{0}^{t} \frac{\left|a^{\prime}(s)\right|\left(2|\xi||\eta|+|\eta|^{2}\right)}{a(s)(|\xi|+|\eta|)^{2}+1} \mathrm{~d} s \leq N \log (1+|\eta|)+C \\
\int_{0}^{t} \frac{\left|a^{\prime}(s)\right| 2|\xi||\eta|}{a(s)(|\xi|+|\eta|)^{2}+1} \mathrm{~d} s \leq N \log (1+|\eta|)+C .
\end{gathered}
$$

Let's pose $|\xi|=r|\eta|$, if $0 \leq r \leq 1$, we have

$$
\int_{0}^{t} \frac{\left|a^{\prime}(s)\right| r|\eta|^{2}}{a(s)(1+r)^{2}|\eta|^{2}+1} \mathrm{~d} s \leq \int_{0}^{t} \frac{\left|a^{\prime}(s)\right||\eta|^{2}}{a(s)|\eta|^{2}+1} \mathrm{~d} s \leq N \log (1+|\eta|)+C .
$$

If $r>1$, we have

$$
\begin{aligned}
\int_{0}^{t} \frac{\left|a^{\prime}(s)\right| r|\eta|^{2}}{a(s)(1+r)^{2}|\eta|^{2}+1} \mathrm{~d} s & \leq \frac{1}{r} \int_{0}^{t} \frac{\left|a^{\prime}(s)\right| r^{2}|\eta|^{2}}{a(s) r^{2}|\eta|^{2}+1} \mathrm{~d} s \leq \\
& \leq \frac{1}{r} N \log (1+r|\eta|)+C \leq N \log (1+|\eta|)+C .
\end{aligned}
$$

This completes the proof of the lemma.

\section{Appendix}

Given $a(t, \xi)=\sum_{i, j}^{1, n} a_{i j}(t) \xi_{i} \xi_{j}$, with $a(t, \xi) \geq 0, a_{i j}(t)=a_{j i}(t)$, $a_{i j}(t) \in C^{1}$, we consider the following condition (due to Kajitani [Ka]): 
On a weakly hyperbolic equation with a term of order zero

there exists a real function $f(t, \xi) \in C\left(0, T ; C^{\infty}\right) \cap C^{1}$ such that

$$
\begin{gathered}
\frac{\left|a_{t}^{\prime}(t, \xi)\right|}{a(t, \xi)+(f(t, \xi))^{2}} \leq f(t, \xi), \\
\int_{0}^{T} f(s, \xi) \mathrm{d} s \leq C \log (\langle\xi\rangle+1), \\
\left|f^{(\alpha)}(t, \xi)\right| \leq C f(t, \xi)\langle\xi\rangle^{-|\alpha|}, \\
\left|f_{t}^{\prime}(t, \xi)\right| \leq C(f(t, \xi))^{2} .
\end{gathered}
$$

We exhibit a function $a(t, \xi)$, analytic in $t$, which doesn't satisfy Kajitani condition.

Let $a(t, \xi)=\left(\xi_{1}-t \xi_{2}\right)^{2}, 0 \leq t \leq 1$. Suppose that $\xi_{2}>0, f \geq 1$ (we add 1 to $f$ if necessary). Define $\xi^{(0)}=\left(1+t \xi_{2}, \xi_{2}\right), \xi^{(1)}=\left(\xi_{2} / 2, \xi_{2}\right)$, then (1) implies that

$$
2 \xi_{2} \leq\left(f+f^{3}\right)\left(t, \xi^{(0)}\right) \leq 2 f^{3}\left(t, \xi^{(0)}\right), \quad f\left(t, \xi^{(0)}\right) \geq \sqrt[3]{\xi_{2}}
$$

and (2) gives

$$
\text { meas }\left\{t: f\left(t, \xi^{(1)}\right)>\frac{1}{m} \sqrt[3]{\xi_{2}}\right\} \frac{1}{m} \sqrt[3]{\xi_{2}} \leq C\left(\log \left\langle\xi^{(1)}\right\rangle+1\right) .
$$

Hence

$$
\text { meas }\left\{t: f\left(t, \xi^{(1)}\right)>\frac{1}{m} \sqrt[3]{\xi_{2}}\right\} \rightarrow 0 \text { as } \xi_{2} \rightarrow+\infty .
$$

In particular for some $\xi_{2}$ big enough there exists $t \in\left[\frac{1}{2}, 1\right]$ such that $f\left(t, \xi^{(1)}\right) \leq(1 / m) \sqrt[3]{\xi_{2}}, f\left(t, \xi^{(0)}\right) \geq \sqrt[3]{\xi_{2}}$. Hence

$$
\begin{aligned}
& \log f\left(t, \xi^{(0)}\right)-\log f\left(t, \xi^{(1)}\right)= \\
& \quad=\frac{\frac{\partial f}{\partial \xi_{1}}\left(t, \xi^{(1)}+\theta\left(\xi^{(0)}-\xi^{(1)}\right)\right)}{f\left(t, \xi^{(1)}+\theta\left(\xi^{(0)}-\xi^{(1)}\right)\right)}\left(1+t \xi_{2}-\frac{1}{2} \xi_{2}\right)
\end{aligned}
$$

with $0<\theta<1$. Since $1+t \xi_{2}-\frac{1}{2} \xi_{2} \leq \sqrt{2\left(1+\xi_{2}^{2}\right)}$, we get the following inequality in the point $\left(t, \xi^{(1)}+\theta\left(\xi^{(0)}-\xi^{(1)}\right)\right)$ :

$$
\sqrt{2}\langle\xi\rangle \frac{\partial f}{\partial \xi_{1}} / f \geq \log m .
$$

It follows that (3) is not satisfied for $\alpha=e_{1}$. 
Nicola Orrù

\section{Acknowledgements}

I would like to thank Prof. Ferruccio Colombini for his help while carrying out the research of this paper.

\section{Bibliography}

[CDS] Colombini (F.), De Giorgi (E.) and Spagnolo (S.) . - Sur les équations hyperboliques avec des coefficients qui ne dépendent que du temps, Ann. Scuola Norm. Sup. Pisa, 6 (1979), pp. 511-559.

[CJS] Colombini (F.), Jannelli (E.) and Spagnolo (S.) .- Well-posedness in the Gevrey classes of the Cauchy problem for a non-strictly hyperbolic equation with coefficients depending on time, Ann. Scuola Norm. Sup. Pisa, 10 (1983), pp. 291-312.

[Hör] HöRmANDeR (L.) .- Linear Partial Differential Operators, Springer, Berlin (1963).

[Ka] KAJITANi (K.) .- The well posed Cauchy problem for hyperbolic operators, Exposé au Séminaire Vaillant (1989).

[Ol] OlEINIK (O.A.) .- On the Cauchy problem for weakly hyperbolic equations of second order, Comm. Pure Appl. Math. 23 (1970), pp. 569-586. 